\title{
Eliminating unnecessary routine head CT scanning in neurologically intact mild traumatic brain injury patients: implementation and evaluation of a new protocol
}

\author{
Seema P. Anandalwar, MD, ${ }^{1}$ Christine Y. Mau, MD, ${ }^{2}$ Chirag G. Gordhan, MD, ${ }^{1}$ Neil Majmundar, MD, ${ }^{2}$ \\ Ahmed Meleis, MD, ${ }^{2}$ Charles J. Prestigiacomo, MD, ${ }^{2}$ and Ziad C. Sifri, MD1 \\ Departments of ${ }^{1}$ Trauma Surgery and ${ }^{2}$ Neurological Surgery, Rutgers New Jersey Medical School, Newark, New Jersey
}

\begin{abstract}
OBJECTIVE The utility of routine repeat head CT (HCT) scans in the management of minimal head injury (MHI) patients with an intracranial hemorrhage $(\mathrm{ICH})$ has been questioned in multiple studies. All these studies analyzed this by obtaining a repeat HCT study, and none examined the effects of eliminating these routine HCT studies in neurologically intact patients. The authors' institution implemented a new "Neurologic Observation without Repeat HCT" (NORH) protocol with no repeat $\mathrm{HCT}$ scanning for patients admitted for $\mathrm{MHI}$ and $\mathrm{ICH}$ whose neurological status was maintained or improved to a Glasgow Coma Scale score of 15 at 24 hours after admission. This purpose of this study was to assess the outcomes and safety of this novel protocol.
\end{abstract}

METHODS Records of patients who sustained blunt trauma MHI and an ICH and/or skull fracture on initial HCT between January 1, 2009, and December 31, 2012, were retrieved from the trauma registry of a Level I trauma center. The authors analyzed 95 patients in whom the NORH protocol was followed. Outcome measures included death, emergency department readmission, neurosurgical intervention, delayed repeat $\mathrm{HCT}$, and length of stay.

RESULTS The NORH protocol was followed for 95 patients; $83 \%$ of the patients were male, the average age was $38 \pm$ 16.0 years old, and the most common cause of trauma was assault $(35 \%)$. Of the 95 patients in whom the NORH protocol was followed, $8(8 \%)$ had a delayed repeat HCT study (> 24 hours) after admission, but none resulted in neurosurgical intervention because of progression of $\mathrm{ICH}$. The average length of stay was $4 \pm 7.2$ days. None of the patients were readmitted to the hospital.

CONCLUSIONS Implementation of the NORH protocol (eliminating routine follow-up HCT) resulted in very low rates of delayed neurological deterioration, no late neurosurgical interventions resulting from ICH progression, very few emergency department revisits, and no readmissions. For a select group of $\mathrm{MHI}$ patients with $\mathrm{ICH}$, the NORH protocol is safe and effective, and can reduce radiation exposure and costs.

http://thejns.org/doi/abs/10.3171/2015.9.JNS151379

KEY WORDS mild traumatic brain injury; computed tomography; repeat head computed tomography

$\mathrm{T}$ HE National Center for Injury Prevention and Control estimates that 1.7 million people sustain a traumatic brain injury (TBI) in the United States each year, ${ }^{6}$ with the vast majority suffering a minimal head injury (MHI), defined as a loss of consciousness and/or retrograde amnesia with a Glasgow Coma Scale (GCS) score greater than $12 .{ }^{11}$ Traditionally, nonoperative management of MHI patients with an intracranial hemorrhage (ICH) has consisted of observation and a routine repeat head CT (HCT) study at 24-48 hours after admission to assess for any ICH evolvement. However, with increasing concern over the use of hospital resources and concern for radiation exposure, the value and necessity of a routine repeat HCT scanning has come into question. ${ }^{3,10,15,16,18,19,21-23,25}$ Despite this, the use of routine repeat HCT studies has actually increased, especially in developed countries. ${ }^{24}$

ABBREVIATIONS GCS = Glasgow Coma Scale; HCT = head CT; ICH = intracranial hemorrhage; LOS = length of stay; $\mathrm{MHI}=$ minimal head injury; NORH = Neurologic Observation without Repeat HCT; TBI = traumatic brain injury.

SUBMITTED June 14, 2015. ACCEPTED September 9, 2015.

INCLUDE WHEN CITING Published online January 15, 2016; DOI: 10.3171/2015.9.JNS151379. 
Some recent studies have suggested that the establishment of a normal neurological status precludes the need for a routine repeat HCT study. ${ }^{2,4,5,9,16-18,22,25}$ In a prospective single-center study, $76 \%$ of patients who suffered an MHI and ICH had a normal neurological status at the time of their repeat HCT study, and none sustained any neurological deterioration or intervention during the hospital course ${ }^{16} \mathrm{~A}$ meta-analysis reported very low intervention rates in patients without evidence of neurological deterioration and argued for the abandonment of routine repeat HCT studies in patients who return to a normal mental status. ${ }^{2}$ Other studies have reported similar outcomes and have reached similar conclusions. ${ }^{2,4,5,22}$ With $75 \%$ of MHI patients regaining a normal mental status after the initial HCT study, a reduction in the number of routine repeat HCT studies in this population has the potential to significantly reduce hospital costs and resource utilization. ${ }^{11}$ However, all published literature details studies, both retrospective and prospective, determining the utility of repeat HCT studies by obtaining one to evaluate progression of ICH and/or need for neurosurgical intervention. $3,10,15,16,18,19,21,22,25$ Furthermore, no studies have reported implementing a protocol to eliminate unnecessary repeat HCT studies to evaluate the safety and efficacy of only obtaining an initial HCT study.

Despite the literature, most institutions continue to obtain routine repeat HCT scans even in neurologically intact patients. Reluctance to practice what is recommended in the literature may be related to concerns over unforeseen risks, such as imminent neurological deterioration. Our institution implemented a selective new Neurologic Observation without Repeat HCT (NORH) protocol in 2009, whereby patients admitted for an MHI and ICH whose neurological status was maintained or improved to a GCS score of 15 at 24 hours after admission do not undergo routine repeat HCT scanning. The indication for repeat HCT in this population was neurological deterioration.

To our knowledge, this study is the first to examine the actual implementation of a protocol eliminating unnecessary routine HCT in neurologically intact MHI patients with ICH. The goal of this study was to assess the safety and efficacy of a protocol such as NORH.

\section{Methods}

Records were retrieved from the trauma registry of adult patients ( $\geq 18$ years) admitted to a Level I trauma center (University Hospital, Newark, NJ) between January 1, 2009, and December 31, 2012, with blunt trauma MHI (GCS score > 12) and an ICH and/or skull fracture on initial HCT. Their records were retrospectively reviewed. This study was approved by the institutional review board at Rutgers New Jersey Medical School. Patient informed consent was not obtained.

\section{Patient Inclusion Criteria}

Patients were included in the study if they were admitted with an MHI and ICH, had a GCS score of 15 at 24 hours after admission, and did not receive a repeat HCT study (NORH protocol). All patients were observed for a minimum of 24 hours based on our previous work. ${ }^{8} \mathrm{~Pa}$ tients who did receive a repeat HCT and had a GCS score of 15 were considered to have deviated from protocol and were analyzed as the comparison group in this analysis. Exclusion criteria included immediate neurosurgical intervention following initial HCT scanning, prior TBI or neurosurgical intervention, associated spinal cord injury, coagulopathy (indicated by an international normalized ratio $>1$ or if the patient was on a regimen of anticoagulation therapy), pregnancy, psychiatric or neurological disorders, or incomplete records. Patients with psychiatric or neurological disorders were excluded because their mental status examination findings can fluctuate due to their disorders and thus be unreliable. Patients with incomplete records as well as those transferred from outside hospitals were excluded because accurate records of initial mental examinations could not be obtained. These patients were all excluded to maintain the accuracy of our study population. Demographics were recorded for each patient and included age, sex, mechanism of injury, Injury Severity Score, and Head Abbreviated Injury Severity score. Patients whose conditions deteriorated and required an emergent repeat HCT study before 24 hours were not considered a deviation from the NORH protocol.

\section{Head Computed Tomography}

All HCT scans were read by a staff radiologist who was on call at the time of the scan and were retrospectively reviewed for data collection. Time to initial HCT study, type of lesion, and comparison with previous HCT scans (same, better, worse) were recorded. Any repeat HCT studies obtained less than 24 hours after admission were considered deviation from the NORH protocol unless clinical indications were present.

\section{Outcome Measures}

Patients were followed for evidence of any repeat HCT scans $>24$ hours after admission (delayed repeat HCT). Delayed repeat HCT scans were ordered based on the attending surgeon's discretion based on clinical concern or documented acute mental status change. Comparisons were made between repeat HCT scans and previous CT scans based on interpretations in the radiology reports and were recorded as same, better, or worse. These patients were monitored for any neurosurgical interventions or change in care based on repeat HCT findings. Neurosurgical intervention was defined as placement of an intracranial pressure monitor or external ventricular drain, or craniotomy. Change in care based on repeat HCT findings was defined as being upgraded to the ICU, administration of mannitol, or intubation. All emergency department visits within 1 year after discharge for TBI-related symptoms were also recorded. Other recorded neurological outcome measures include length of stay (LOS), GCS score at discharge, and disposition at discharge (home vs rehabilitation center).

\section{Statistical Analysis}

Descriptive analysis was conducted using Microsoft Excel (Microsoft Corp.). Wilcoxon rank-sum tests com- 
paring medians, Fisher's exact tests, and independent ttests were performed using SAS (SAS Institute Inc.). Statistical significance was defined as $\mathrm{p}<0.05$.

\section{Results}

During the 48-month study period, 533 patients were admitted with an MHI and ICH at our institution. Of these patients, $370(69 \%)$ were excluded for coagulopathy, history of TBI, immediate neurosurgical intervention, transfer from an outside hospital, or incomplete records. Twentyone patients $(3.9 \%)$ had neurological deterioration less than 24 hours from admission and were excluded from the protocol and this study. Of the remaining 142 eligible patients, treatment in 47 patients $(33 \%)$ was considered a deviation from protocol; these patients constituted the comparison group (GCS score of 15 at 24 hours with a repeat HCT), and the remaining $95(67 \%)$ patients were treated according to the NORH protocol and are included in this analysis (Fig. 1).

Of the 95 patients, $83 \%$ were male and the average age was $38 \pm 16.0$ years old. The most common cause of trauma was assault (35\%) followed by fall (34\%). A majority of patients were admitted with a GCS score of $15(80 \%)$, and $86 \%$ were admitted to the progressive care unit from the emergency department. The most common lesion on initial HCT was a subdural hemorrhage (72\%), and 61\% had multiple lesions (Table 1).

To ensure that there was no selection bias among the patients in whom the protocol was followed versus those in whom it was not, the 47 patients who received repeat HCT within 24 hours of initial HCT scanning even without neurological deterioration were compared with the study population. There was no significant difference in age, lesion on initial HCT scanning, LOS, and number of patients with acute mental status change. Of these 47 patients, $1(2.1 \%)$ patient had a medical intervention after the repeat HCT study. This patient did not suffer a neurological decline or have any change in ICH. The patient's repeat HCT revealed a venous sinus thrombus for which the patient received aggressive intravenous hydration therapy. The 47 patients in whom treatment deviated from the NORH protocol had a significantly greater number of delayed repeat HCT studies $(23 \%$ vs $8 \%, \mathrm{p}=0.014)$ and number of emergency department revisits $(15 \%$ vs $3 \%, \mathrm{p}=$ 0.005 ) compared with the study population.

Of the 95 patients in whom the NORH protocol was followed, 8 (8.4\%) had a delayed repeat HCT study $(>24$ hours) after admission. Of these 8 patients, 5 (63\%) HCT studies were ordered based the attending surgeon's clinical judgment and $3(38 \%)$ were ordered based on a documented acute mental status change. None of the 8 repeat HCT studies led to an intervention for progression of an ICH.

Of the 5 patients with a delayed repeat HCT study due to clinical judgment, none (0\%) had any change in $\mathrm{ICH}$, and 1 patient (20\%) had a neurosurgical intervention for a condition unrelated to the presence of the ICH. This particular patient was taken to the operating room for concern of right frontal sinus obstruction and extraocular muscle impingement. Of the patients with a delayed repeat HCT study due to acute mental status change, all $3(100 \%)$ had worsened CT findings. None of these patients had any neurosurgical intervention based on the repeat HCT study findings or for progression of the ICH. Average time to an acute mental status change was $70 \pm 9.2$ hours after admission. Two of three patients were upgraded to the ICU, one for increased agitation and the other for respiratory decline (Table 2). Both were receiving seizure prophylaxis and one was later intubated during the ICU stay.

Of the total study population, only $3(3.2 \%)$ returned to the emergency department for TBI-related symptoms. All 3 patients underwent repeat HCT in the emergency de-

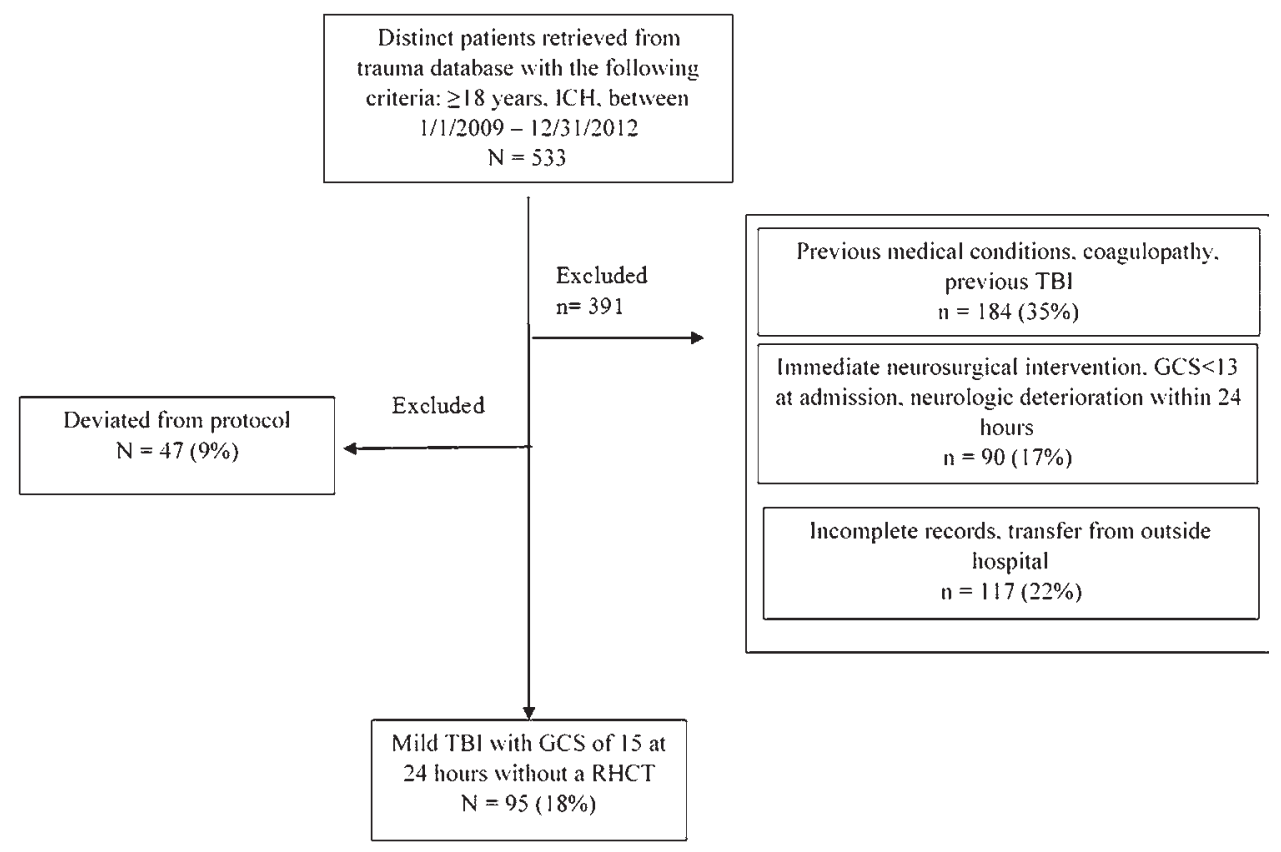

FIG. 1. Study population included and excluded from the NORH protocol. RHCT = repeat HCT. 
TABLE 1. Patient demographics of the entire study population and those in the delayed repeat HCT group*

\begin{tabular}{|c|c|c|c|}
\hline Variable & $\begin{array}{l}\text { Study Population } \\
\qquad(\mathrm{n}=95)\end{array}$ & $\begin{array}{l}\text { Delayed RHCT } \\
\quad(n=8) \dagger\end{array}$ & $\begin{array}{c}\mathrm{p} \\
\text { Value }\end{array}$ \\
\hline Mean age, yrs & $38 \pm 16.0$ & $43.5 \pm 18.2$ & 0.48 \\
\hline Male sex & $79(83.2)$ & $8(100.0)$ & 0.35 \\
\hline $\begin{array}{l}\text { Type of trauma } \\
\text { MVC } \\
\text { Fall } \\
\text { Pedestrian struck } \\
\text { Assault } \\
\text { Unknown }\end{array}$ & $\begin{array}{c}19(20.0) \\
32(33.7) \\
9(9.5) \\
33(34.7) \\
2(2.1)\end{array}$ & $\begin{array}{l}0(0.0) \\
3(37.5) \\
1(12.5) \\
4(50.0) \\
0(0.0)\end{array}$ & 0.58 \\
\hline Mean ISS & $21.0 \pm 5.9$ & $25.1 \pm 5.6$ & 0.08 \\
\hline $\begin{array}{l}\text { GCS score on arrival } \\
13 \\
14 \\
15\end{array}$ & $\begin{array}{c}4(4.2) \\
15(15.8) \\
76(80.0)\end{array}$ & $\begin{array}{l}1(12.5) \\
2(25.0) \\
5(62.5)\end{array}$ & 0.31 \\
\hline $\begin{array}{l}\text { ED disposition } \\
\text { ICU } \\
\text { PCU } \\
\text { Floor }\end{array}$ & $\begin{array}{c}10(10.5) \\
82(86.3) \\
3(3.2)\end{array}$ & $\begin{array}{l}1(12.5) \\
7(87.5) \\
0(0.0)\end{array}$ & 1.00 \\
\hline $\begin{array}{l}\text { Mean time from arrival to } \\
\text { 1st HCT, hrs }\end{array}$ & $1.0 \pm 1.3$ & $1.7 \pm 2.6$ & 0.48 \\
\hline \multicolumn{4}{|l|}{ Type of lesion $\ddagger$} \\
\hline $\mathrm{SDH}$ & $68(72.0)$ & $8(100.0)$ & 0.11 \\
\hline IPH & $21(22.0)$ & $4(50.0)$ & 0.10 \\
\hline $\mathrm{SAH}$ & $33(35.0)$ & $5(62.5)$ & 0.14 \\
\hline Skull fracture & $41(43.0)$ & $6(75.0)$ & 0.14 \\
\hline $\mathrm{EH}$ & $14(15.0)$ & $1(12.5)$ & 1.00 \\
\hline IVH & $1(1.0)$ & $0(0.0)$ & 1.00 \\
\hline
\end{tabular}

$\mathrm{ED}=$ emergency department; $\mathrm{EH}=$ epidural hematoma; $\mathrm{IPH}=$ intraparenchymal hemorrhage; ISS = Injury Severity Score; IVH = intraventricular hemorrhage; $\mathrm{MVC}=$ motor vehicle crash; $\mathrm{PCU}=$ progressive care unit; $\mathrm{RHCT}$ = repeat $\mathrm{HCT} ; \mathrm{SAH}$ = subarachnoid hemorrhage; $\mathrm{SDH}$ = subdural hemorrhage.

* Values are presented as the number of patients (\%) unless indicated otherwise. Mean values are presented as the mean \pm SD.

$\dagger$ Patients in this category were managed according to the protocol and underwent repeat HCT more than 24 hours after admission because of clinical concern.

$\ddagger$ Patients might have had more than 1 type of lesion reported on the initial HCT study.

partment, of whom 2 (66.7\%) showed improvement in ICH and $1(33.3 \%)$ showed worsening, but none were admitted to the hospital from the emergency department. There was no significant difference in postdischarge emergency department visits in patients in whom the NORH protocol was followed between the patients who did not undergo delayed HCT scanning and those who did (3\% vs $13 \%$, p $=0.25)($ Table 3$)$.

\section{Discussion}

To our knowledge, this is the first study to analyze the safety and efficacy of the NORH protocol to eliminate repeat HCT scanning in MHI patients with ICH who remained neurologically intact at 24 hours. This analysis revealed that the NORH protocol, if implemented for a select group of patients, is safe and effective. There were very low rates of delayed neurological deterioration, no late interventions for ICH progression, very few emergency department revisits, and no readmissions for TBI sequelae.

Of the patients in the NORH protocol, 8 patients $(8 \%)$ underwent delayed repeat HCT ( $>24$ hours after admission). Only 3 of these 8 patients (38\%) demonstrated worsening of their $\mathrm{ICH}$, and none resulted in neurosurgical intervention, indicating that risk of a missed intervention or significant progression of an ICH due to a lack of a routine repeat HCT study is very low. The average time to repeat HCT in the 3 patients with worsening ICH was 70 hours after admission. Homnick et al. ${ }^{8}$ reported that the ICH stops progressing by 24 hours in $97 \%$ of MHI patients, which is supported by a prospective study showing early progression for all levels of TBI-related ICH.12 Patients whose conditions deteriorated within 24 hours and received an emergent repeat HCT were excluded from this study. The majority $(92 \%)$ of the patients in whom the NORH protocol was followed only received a single HCT study. Of the few who received delayed repeat HCT scans, the majority showed either no change or an improvement of the $\mathrm{ICH}$, and none required any neurosurgical intervention.

The results of this NORH protocol implementation support previously reported studies that routine repeat HCT studies in MHI patients are not only unnecessary but also a resource utilization concern. Three prospective studies ${ }^{4,5,16}$ and several retrospective studies ${ }^{2,9,17,18,22,25}$ have demonstrated little need for routine repeat HCT studies in MHI patients who remain neurologically stable. A recent meta-analysis by Almenawer et al. ${ }^{2}$ found that the greatest predictive factor of neurosurgical intervention after a repeat HCT is preceding acute neurological deterioration. In that study, the pooled intervention rate after a routine repeat HCT study in neurologically stable patients was $0.6 \%$ with a significantly greater intervention rate in those who suffered neurological decline. Our study demonstrated similar results; no patient in whom the NORH protocol was followed and who remained neurologically stable required neurosurgical intervention. The only pa-

TABLE 2. Patients with an acute neurological decline after 24 hours on the NORH protocol

\begin{tabular}{|c|c|c|c|c|c|c|c|c|c|}
\hline $\begin{array}{l}\text { Case } \\
\text { No. }\end{array}$ & $\begin{array}{l}\text { Age } \\
\text { (yrs) }\end{array}$ & $\begin{array}{l}\text { GCS Score } \\
\text { on Arrival }\end{array}$ & Initial HCT Findings & $\begin{array}{c}\text { Time to } \\
\text { Decline (hrs) }\end{array}$ & $\begin{array}{l}\text { No. of } \\
\text { RHCTs }\end{array}$ & Findings on RHCTs & $\begin{array}{l}\text { Upgrade } \\
\text { of Care }\end{array}$ & $\begin{array}{l}\text { Neurosurgical } \\
\text { Intervention }\end{array}$ & $\begin{array}{c}\text { ED } \\
\text { Revisit }\end{array}$ \\
\hline 1 & 55 & 15 & $\mathrm{SDH}, \mathrm{SAH}$ & 78 & 1 & $\begin{array}{l}\text { Worsened: prefrontal lobe } \\
\text { IPH \& worsening edema }\end{array}$ & Yes & No & No \\
\hline 2 & 54 & 15 & $\mathrm{SDH}, \mathrm{IPH}, \mathrm{SAH}, \mathrm{EH}$ & 72 & 1 & Worsened: increased SDH & No & No & No \\
\hline 3 & 66 & 15 & SDH, IPH, skull fracture & 60 & 3 & Worsened: new SAH \& SDH* & Yes & No & No \\
\hline
\end{tabular}

* Two additional repeat CT studies were performed. 
TABLE 3. Comparing outcomes between single HCT and delayed RHCT patients*

\begin{tabular}{lccc}
\hline \multicolumn{1}{c}{ Variable } & $\begin{array}{c}\text { Single HCT } \\
(n=87)\end{array}$ & $\begin{array}{c}\text { Delayed } \\
\text { RHCT }(n=8)\end{array}$ & $\begin{array}{c}p \\
\text { Value }\end{array}$ \\
\hline Mean LOS, days & $4.0 \pm 7.2$ & $14.0 \pm 13.4$ & 0.074 \\
\hline Mean GCS score at discharge & $15.0 \pm 0.11$ & $15.0 \pm 0.0$ & 0.32 \\
\hline $\begin{array}{l}\text { Disposition } \\
\text { Home }\end{array}$ & $83(95.4)$ & $6(75.0)$ & 0.04 \\
$\quad$ Rehabilitation center & $2(2.3)$ & $2(25.0)$ & \\
\hline Return to ED & $2(3.0)$ & $1(12.5)$ & 0.25 \\
\hline
\end{tabular}

* Values represent the number of patients (\%) unless indicated otherwise. Mean values are presented as the mean \pm SD.

tients to experience any change in care were those who received a delayed repeat HCT study (HCT $>24$ hours) as a result of a delayed (70 \pm 9 hours) acute neurological decline. The intervention rate in those in whom the NORH protocol was followed was $0.2 \%$, which is comparable to the pooled rate reported in the meta-analysis. Only 1 patient had a neurosurgical intervention following a delayed repeat HCT; however, the cause was not for progression of the ICH but for progressive obstruction of the frontal sinuses. Therefore, there were no neurosurgical interventions undertaken for progression of an ICH. This very low intervention rate is influenced by patient selection and sample size, but it validates low intervention rates for patients who remain neurologically stable. Despite these studies, there is still fear of the unknown in not obtaining a repeat HCT study. This reflects clinical practice across most institutions where attending physicians are hesitant to implement an NORH protocol. This may be attributed to a variety of reasons, including controversy in the literature and the long-standing tradition of a nonoperative management protocol, but the lack of a published study demonstrating the safety of only performing an initial HCT study may be a factor.

Literature is equivocal, and studies recommending the discontinuation of repeat HCT studies still perform routine repeat HCT to confirm lack of ICH progression. ${ }^{2,4,5,9,16-18,22,25}$ Several studies have argued that there is a benefit in performing routine repeat HCT scanning in MHI patients. ${ }^{3,714,19-21}$ This reflects the clinical practice of most institutions where routine repeat HCT studies are still part of the standard protocol for nonoperative management of MHI patients. Thorson et al..$^{21}$ found that $30 \%$ of routine repeat HCT studies demonstrated injury progression. Of the patients who required operative management, up to $59 \%$ had no clinical decline prior to their CT study. Bee et al. ${ }^{3}$ reported similar results, with $28 \%$ of patients who underwent routine repeat HCT exhibiting worsened CT findings; $9 \%$ of their study population required neurosurgical intervention. Several factors might explain the variation in findings as well as highlight an important emphasis in our NORH protocol. The threshold to operate varies among institutions. Bee et al. ${ }^{3}$ and Thorson et al. ${ }^{21}$ reported that $28 \%$ and $32 \%$ of their surgically treated patients, respectively, had no associated clinical deterioration, and the decision for surgery was based solely on CT findings. Our difference in neurosurgery rates, and those noted in the literature, might be due to varying thresholds for operative intervention. Furthermore, many of the studies that argue for routine repeat HCT studies include patients receiving long-term anticoagulation therapy. It should be reiterated that our NORH protocol exclusively applies to patients who have no evidence of a coagulopathy, either by medical history or laboratory results. In addition, these studies vary in timing of routine repeat HCT; Thorson et al. ${ }^{21}$ reported that more than half of their patients underwent repeat HCT scanning in less than 6 hours, while other studies have quoted a range of 12-24 hours. Any patient mentioned in these studies whose condition deteriorated prior to 24 hours of observation would have received an emergent HCT and would have been excluded from the NORH protocol. We believe that it is the careful selection of the patients who qualify for the protocol that contributes to the safety and outcomes reported in this analysis.

This study found that 47 of the 142 eligible patients (33\%) deviated from the NORH protocol (GCS score of 15 at 24 hours and a routine repeat HCT). To ensure that there was no selection bias and the severity of injury did not dictate who deviated from protocol, all TBI-related factors were compared between the patients in whom management deviated from protocol and the study population. None of the analyses found a significant difference in age, type of lesion on initial CT, admission GCS score, or type of trauma between the 2 cohorts, demonstrating lack of selection bias. Within the deviation from protocol group, only 1 patient received medical intervention consisting of aggressive intravenous hydration for a venous sinus thrombus. The reason for this $33 \%$ rate of deviation from the single HCT protocol is unclear and is not well documented. Possibilities include the lack of documentation of acute neurological changes, repeat HCT due to a need for general anesthesia for other operations, and questionable ICH on initial HCT. Although $34 \%$ of the patients in whom management deviated from the protocol had worsened repeat HCT findings (routine repeat HCT < 24 hours), this was comparable to rates reported in other studies. ${ }^{2}$ Furthermore, all patients had a GCS score of 15 at 24 hours, and $98 \%$ of those with worsened HCT findings resolved without neurosurgical intervention. Thus, if the NORH protocol had been followed, the majority of patients would have had favorable outcomes. With this in mind, we propose the following decision-making algorithm for MHI patients to safely reduce resource utilization and hospital costs (Fig. 2).

Sifri et al. ${ }^{18}$ reported that serial neurological examinations to identify those in need of intervention have a negative predictive value of $100 \%$. All patients in our study population were managed with serial neurological examinations and had established normal neurological examination findings (GCS Score 15) by 24 hours. These patients were followed for the remainder of their admission with serial neurological assessments to identify any acute change. Of the delayed repeat HCT studies performed in 8 patients, 5 studies were nonemergent (ordered due to attending request) and 3 were considered emergent (ordered because of a documented acute mental status change). The only patients in whom worsening was revealed on CT 


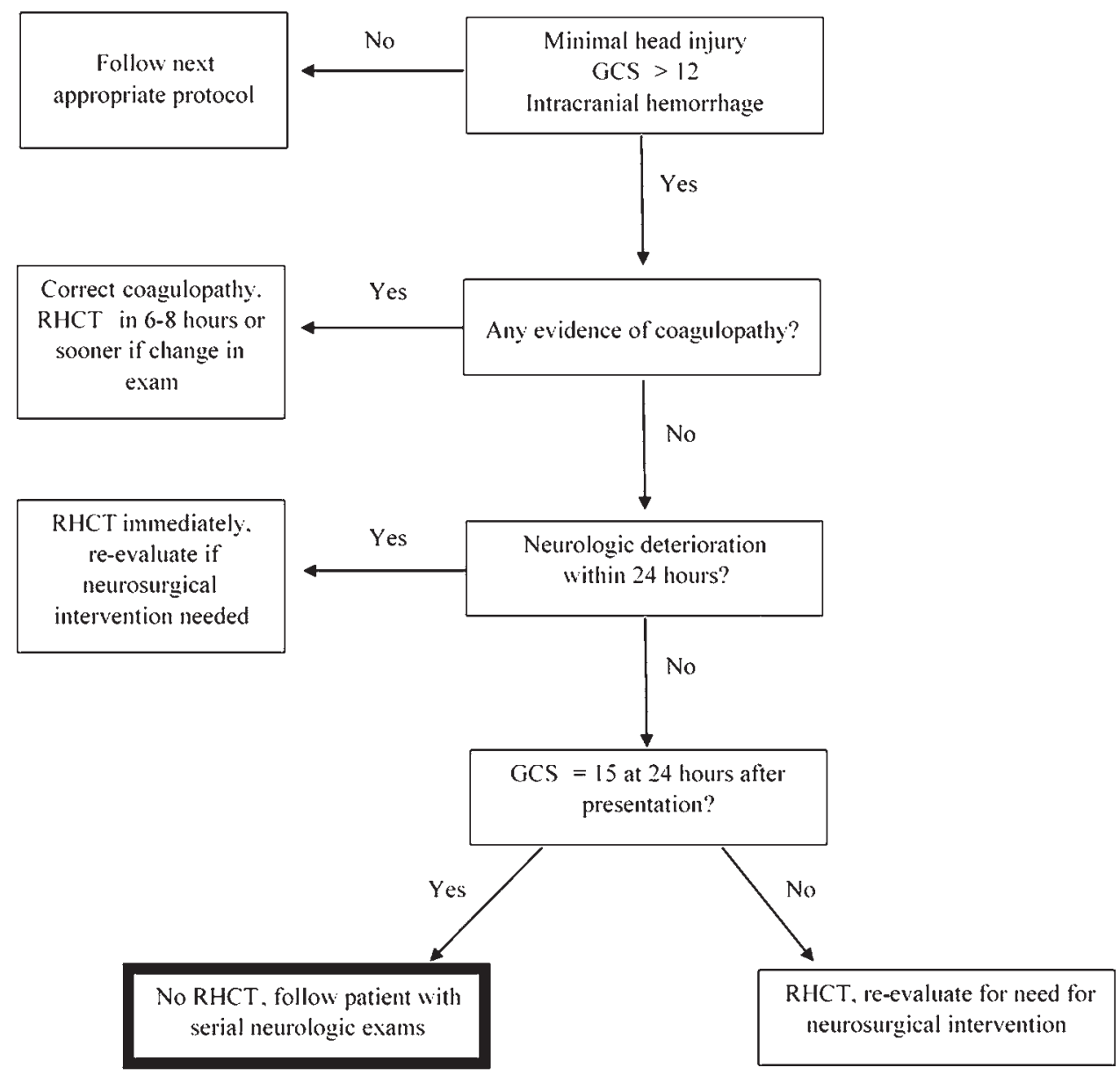

FIG. 2. Decision-making algorithm for repeat $\mathrm{HCT}$ in patients who present with $\mathrm{MHI}$ and $\mathrm{ICH}$.

scanning were those who demonstrated a change in mental status, supporting the idea that patients can be adequately identified for ICH progression by neurological examination.

Stein et al. ${ }^{19}$ reported a significant decrease in cost in MHI patients who underwent a repeat HCT study only after deterioration versus those who underwent routine repeat HCT (\$1321 vs \$1563). They also reported that not obtaining a routine repeat HCT study could be less effective if it resulted in a delayed intervention leading to coma or disability. We believe, however, that with careful selection of patients (those who return to a normal neurological status) it is possible to reduce chances of missing a progression. Based on our data, in a select population undergoing this NORH protocol, we had no deaths, no long-term morbidity, and no delayed neurosurgical interventions. Additionally, the need to follow up with routine repeat HCT studies has been found to be associated with a greater number of total HCT scans and a longer LOS. ${ }^{1}$ With a greater number of HCT scans, patients are exposed unnecessarily to additional radiation, which is of particular concern in the pediatric population. The cost associated with additional HCT lies not only in the actual cost of the scan, but the time taken by the attending radiologist, as well as the attending surgeons to evaluate whether an intervention needs to occur. These unnecessary scans also contribute to longer LOS. ${ }^{1}$ One study estimates that a single night in the ICU costs $\$ 2575$ vs $\$ 1488 .{ }^{13}$ Excessive radiation exposure and unnecessary resource utilization further emphasize the need for an NORH protocol.

\section{Limitations}

There are several limitations to this study. This is a retrospective single-center analysis limited by a moderate sample size. CT readings were not blinded, and data collection was limited to the information provided in the medical records. Reasons for protocol deviation were not well documented and were left to the discretion of the attending surgeon. In addition, ordering of a delayed repeat HCT study was not standardized and the indication was not always documented. Although there were patients in whom management deviated from the protocol, no selection bias was identified, and if the NORH protocol had been followed, the patients would have had favorable outcomes.

Despite these limitations, this is the first study to document the safety of an NORH protocol for MHI and ICH patients. After the completion of this study, our institution continues to follow the NORH protocol successfully with good outcomes. An NORH protocol, if implemented in similar trauma centers, can be safe, cost-effective, and has the potential to reduce the hospital burden attributed to this high-incidence disease. 


\section{Conclusions}

To our knowledge, this is the first study that examined the safety and efficacy of the implementation of a protocol that eliminated routine repeat HCT studies on neurologically intact patients and assessed the outcomes. The NORH protocol was found to be safe for patients with few delayed HCT studies, no neurosurgical intervention, few emergency department revisits, and no readmissions in our enrolled patients.

An NORH protocol in MHI patients with ICH and return of GCS score to 15 at 24 hours reduces radiation exposure, costs, and resource utilization and is a potential benefit for all involved stakeholders. Prospective studies with actual implementation of a similar protocol need to be done for further study.

\section{References}

1. AbdelFattah KR, Eastman AL, Aldy KN, Wolf SE, Minei JP, Scott WW, et al: A prospective evaluation of the use of routine repeat cranial CT scans in patients with intracranial hemorrhage and GCS score of 13 to 15 . J Trauma Acute Care Surg 73:685-688, 2012

2. Almenawer SA, Bogza I, Yarascavitch B, Sne N, Farrokhyar F, Murty N, et al: The value of scheduled repeat cranial computed tomography after mild head injury: single-center series and meta-analysis. Neurosurgery 72:56-64, 2013

3. Bee TK, Magnotti LJ, Croce MA, Maish GO, Minard G, Schroeppel TJ, et al: Necessity of repeat head CT and ICU monitoring in patients with minimal brain injury. J Trauma 66:1015-1018, 2009

4. Brown CV, Weng J, Oh D, Salim A, Kasotakis G, Demetriades D, et al: Does routine serial computed tomography of the head influence management of traumatic brain injury? A prospective evaluation. J Trauma 57:939-943, 2004

5. Brown CV, Zada G, Salim A, Inaba K, Kasotakis G, Hadjizacharia $P$, et al: Indications for routine repeat head computed tomography (CT) stratified by severity of traumatic brain injury. J Trauma 62:1339-1345, 2007

6. Bruns J Jr, Hauser WA: The epidemiology of traumatic brain injury: a review. Epilepsia 44 (Suppl 10):2-10, 2003

7. Ding J, Yuan F, Guo Y, Chen SW, Gao WW, Wang G, et al: A prospective clinical study of routine repeat computed tomography (CT) after traumatic brain injury (TBI). Brain Inj 26:1211-1216, 2012

8. Homnick A, Sifri Z, Yonclas P, Mohr A, Livingston D: The temporal course of intracranial haemorrhage progression: how long is observation necessary? Injury 43:2122-2125, 2012

9. Huynh T, Jacobs DG, Dix S, Sing RF, Miles WS, Thomason MH: Utility of neurosurgical consultation for mild traumatic brain injury. Am Surg 72:1162-1167, 2006

10. Kaups KL, Davis JW, Parks SN: Routinely repeated computed tomography after blunt head trauma: does it benefit patients? J Trauma 56:475-481, 2004

11. Levin HS: Outcome from mild head injury, in Narayan RK, Wilberger JE Jr, Povlishock JT (eds): Neurotrauma. New York: McGraw-Hill, 1996, pp 749-754

12. Narayan RK, Maas AI, Servadei F, Skolnick BE, Tillinger MN, Marshall LF: Progression of traumatic intracerebral hemorrhage: a prospective observational study. J Neurotrauma 25:629-639, 2008

13. Nishijima DK, Sena MJ, Holmes JF: Identification of lowrisk patients with traumatic brain injury and intracranial hemorrhage who do not need intensive care unit admission. J Trauma 70:E101-E107, 2011
14. Park HK, Joo WI, Chough CK, Cho CB, Lee KJ, Rha HK: The clinical efficacy of repeat brain computed tomography in patients with traumatic intracranial haemorrhage within 24 hours after blunt head injury. Br J Neurosurg 23:617-621, 2009

15. Sharifuddin A, Adnan J, Ghani AR, Abdullah JM: The role of repeat head computed tomography in the management of mild traumatic brain injury patients with a positive initial head CT. Med J Malaysia 67:305-308, 2012

16. Sifri ZC, Homnick AT, Vaynman A, Lavery R, Liao W, Mohr A, et al: A prospective evaluation of the value of repeat cranial computed tomography in patients with minimal head injury and an intracranial bleed. J Trauma 61:862-867, 2006

17. Sifri ZC, Livingston DH, Lavery RF, Homnick AT, Mosenthal AC, Mohr AM, et al: Value of repeat cranial computed axial tomography scanning in patients with minimal head injury. Am J Surg 187:338-342, 2004

18. Sifri ZC, Nayak N, Homnick AT, Mohr AA, Yonclas P, Livingston DH: Utility of repeat head computed tomography in patients with an abnormal neurologic examination after minimal head injury. J Trauma 71:1605-1610, 2011 (Erratum in J Trauma 72:538, 2012)

19. Stein SC, Fabbri A, Servadei F: Routine serial computed tomographic scans in mild traumatic brain injury: when are they cost-effective? J Trauma 65:66-72, 2008

20. Thomas BW, Mejia VA, Maxwell RA, Dart BW, Smith PW, Gallagher MR, et al: Scheduled repeat CT scanning for traumatic brain injury remains important in assessing head injury progression. J Am Coll Surg 210:824-832, 2010

21. Thorson CM, Van Haren RM, Otero CA, Guarch GA, Curia E, Barrera JM, et al: Repeat head computed tomography after minimal brain injury identifies the need for craniotomy in the absence of neurologic change. J Trauma Acute Care Surg 74:967-965, 2013

22. Velmahos GC, Gervasini A, Petrovick L, Dorer DJ, Doran ME, Spaniolas K, et al: Routine repeat head CT for minimal head injury is unnecessary. J Trauma 60:494-501, 2006

23. Vos PE, Alekseenko Y, Battistin L, Ehler E, Gerstenbrand F, Muresanu DF, et al: Mild traumatic brain injury. Eur J Neurol 19:191-198, 2012

24. Wang MC, Linnau KF, Tirschwell DL, Hollingworth W: Utility of repeat head computed tomography after blunt head trauma: a systematic review. J Trauma 61:226-233, 2006

25. Washington CW, Grubb RL Jr: Are routine repeat imaging and intensive care unit admission necessary in mild traumatic brain injury? J Neurosurg 116:549-557, 2012

\section{Disclosures}

The authors report no conflict of interest concerning the materials or methods used in this study or the findings specified in this paper.

\section{Author Contributions}

Conception and design: Sifri. Acquisition of data: Anandalwar. Analysis and interpretation of data: Sifri, Anandalwar, Mau, Gordhan, Majmundar, Meleis. Drafting the article: Anandalwar, Mau. Critically revising the article: Sifri, Mau, Prestigiacomo. Reviewed submitted version of manuscript: Sifri, Anandalwar, Mau, Prestigiacomo. Approved the final version of the manuscript on behalf of all authors: Sifri. Statistical analysis: Anandalwar. Study supervision: Sifri.

\section{Correspondence}

Ziad C. Sifri, Division of Trauma and Critical Care, New Jersey Trauma Center, 150 Bergen St., M232, Newark, NJ 07101. email: sifrizi@njms.rutgers.edu. 